\title{
The prognostic factors of resected non-small cell lung cancer with chest wall invasion
}

\author{
Chang Young Lee', Chun Sung Byun', Jin Gu Lee', Dae Joon Kim, Byoung Chul Cho², Kyung Young Chung ${ }^{1}$ and \\ In Kyu Park ${ }^{*}$
}

\begin{abstract}
Background: We retrospectively reviewed the clinical features and surgical outcomes of patients with a surgically resected NSCLC invading chest wall in order to identify prognostic factors that impact long term survival.

Methods: Between January 1990 and December 2009, 107 patients who underwent surgical resection for chest wall invading NSCLC were reviewed. Tumors invading only the parietal pleura were defined as superficial invasions, and those involving the soft tissue or ribs were defined as deep invasions.

Results: There were 91 men and 16 women; median age was 64 years (range 30 to 80 years). Overall 5 year survival rate was $26.3 \%$. The univariate prognostic factors for survival included gender, extent of resection (pneumonectomy vs lobectomy), tumor size (> $5 \mathrm{~cm}$ vs $\leq 5 \mathrm{~cm}$ ), nodal status (N0 or N1 vs N2), completeness of resection (complete vs incomplete) and completeness of adjuvant chemotherapy. At multivariate analysis, five independent prognostic factors were shown; depth of invasion (superficial vs deep), tumor size, nodal status, completeness of resection, and completeness of adjuvant chemotherapy. In patients with completely resected T3NO NSCLC, completion of chemotherapy is the only prognostic factor for long term survival.
\end{abstract}

Conclusions: Completeness of resection, nodal status, depth of invasion, tumor size, and adjuvant chemotherapy were prognostic factors for long-term survival in NSCLC patients with chest wall invasion. Because of poor prognosis in cases with chest wall invasion that have N2 positive LN, that is difficult to achieve complete resection and that need pneumonectomy, definite chemoradiotherapy or neoadjuvant chemoradiotherapy should be considered first in these cases.

Keywords: Chest wall, lung cancer, prognosis, adjuvant chemotherapy

\section{Background}

Although incomplete resection and the presence of nodal involvement, especially in the $\mathrm{N} 2$ station, have been consistently reported as poor prognostic factors of non-small cell lung cancer with chest wall invasion, other factors influencing survival are still unclear [1-11].

Whether adjuvant chemotherapy or radiotherapy is mandatory for patients with completely resected chest wall invading NSCLC without nodal involvement remains under debate $[2,5,6,12,13]$.

\footnotetext{
* Correspondence: ikpark@snu.ac.kr

${ }^{3}$ Department of Thoracic and Cardiovascular Surgery, Seoul National

University Hospital, Seoul National University College of Medicine

Full list of author information is available at the end of the article
}

Also, there are suggestions that the depth of chest wall invasion may influence prognosis following resection of lung cancer [11].

Herein, we retrospectively reviewed the clinical features and surgical outcomes of patients with a surgically resected NSCLC invading chest wall in order to identify prognostic factors that impact long term survival and to suggest the optimal treatment strategy according to these prognostic factors.

\section{Materials and methods Patients}

Between January 1990 and December 2009, 107 patients with chest wall invading NSCLC were surgically treated at our institution. Patient records were obtained from a database that contained prospectively collected data

\section{Biomed Central}


cohort for any patient undergoing surgery for lung malignancies at our departments. Among 2,138 patients in our data cohort, 282 patients with pT3 NSCLC were selected. Patients with a T3 tumor in the invasion of mediastinal pleura, diaphragm, or the pericardium were excluded. Also, patients with tumor close to main bronchus $(<2 \mathrm{~cm})$ were not included. Patients who received any type of preoperative chemotherapy or radiotherapy, or who underwent wedge resection or segmentectomy, were not selected. However, the subset of patients with T3 superior sulcus tumor included in this study had a chest wall invasion confined only to the parietal pleura or the soft tissue in thorax, which was completely removed through a single incision.

The charts and pathologic report of 107 patients were reviewed. Demographic data and first presenting symptoms were collected. The preoperative evaluation included a physical examination, routine blood tests, electrocardiography, pulmonary function test, and a lung perfusion scan if necessary. The routine staging workup included a chest computed tomography (CT) scan, abdomen CT scan or ultrasonography and fiberoptic bronchoscopy. Bone scintigraphy was routinely performed before 2007 in 104 patients. Fluorodeoxyglucose positron emission tomography (FDG-PET) was performed in 14 patients since 2003. Mediastinoscopy was selectively performed in 28 patients when it was suspected that the patient had enlarged mediastinal lymph nodes on the CT scan or PET scan. Cancers were staged according to the the $6^{\text {th }}$ edition of TNM Classification of Malignant Tumors.

\section{Surgical technique and pathologic examination}

In all patients including those with a superior sulcus tumor except for one patient, posterolateral thoracotomy was performed. Systematic lymph node dissection was completed in all patients.

When chest wall invasion was preoperatively suspected, a chest wall resection including the affected rib was scheduled. Extrapleural resection was performed when the parietal pleura could be easily freed from the chest wall. In case of intraoperative evidence of deep parietal invasion, a decision on whether a chest wall resection or a wide extrapleural resection with involved soft tissue should be performed was determined according to each surgeon's decision. In these cases, resection margin was confirmed intraoperatively through the frozen section for complete resection. Pneumonectomy was performed whenever the mass crossed over the major fissure.

In a pathological examination of the resected specimens, tumors invading only the parietal pleura were defined as superficial invasions, and those involving the soft tissue or ribs were defined as deep invasions.

A complete (R0) resection was defined as a pathologic demonstration of negative tissue margins. Patients who had a complete gross resection in the thoracotomy but were found to have positive margins or extracapsular invasion of retrieved $\mathrm{LN}$ on the final pathologic review were classified as having undergone microscopically incomplete (R1) resections. A gross residual disease after attempted resection was classified as $\mathrm{R} 2$.

\section{Adjuvant treatment}

There was no consistent institutional protocol with regard to adjuvant treatment for patient with surgically resected NSCLC invading the chest wall. Adjuvant treatment was determined by each surgeon and the referred oncologist. Three or more cycles of platinum based chemotherapy were regarded as a completion of chemotherapy. When radiotherapy was performed, it consisted of parietal radiotherapy in cases of stage IIB diseases, and parietal and mediastinal radiotherapy in cases of stage IIIA diseases.

\section{Recurrences and survival}

A recurrence of the ipsilateral chest wall, lung, pleura, mediastinum, or cervical lymph node was defined as locoregional recurrences. The remaining recurrences were defined as systemic recurrences.

Survival was calculated from the date of surgery until death or the date of the last follow-up. Survival rates, including postoperative and non-cancer related deaths, were calculated by the Kaplan-Meier method and compared using the log-rank test. Percentage comparisons were made by the $\chi^{2}$ test. Univariate and multivariate analysis were performed using the Cox proportional hazards regression model to determine factors potentially predicting survival. Factors identified at $\mathrm{p}<0.20$ by univariate analysis and known prognostic factors such as age and radiotherapy were selected for inclusion in a multivariate Cox proportional hazards regression model. All statistical analyses were performed using the SPSS 12.0 software package (SPSS, Chicago, Illinois). Results were considered significant if the $p$ value was less than 0.05 .

\section{Results}

\section{Demographics}

There were 91 men and 16 women; the median age was 64 years (range 30 to 80 years). One or more of the following symptoms were present in the 96 patients: chest pain $(\mathrm{n}=45,41 \%)$, cough $(\mathrm{n}=47,44 \%)$, hemoptysis or blood tinged sputum $(\mathrm{n}=19,18 \%)$, and dyspnea $(\mathrm{n}=7$, $7 \%)$. Eleven patients (10\%) were asymptomatic. Seventy one $(66 \%)$ patients had a history of smoking. The mean FEV1 was $77 \%$ of the predicted value (range 22 to $133 \%$ ). A superior sulcus tumor occurred in 17 (16\%) patients.

\section{Surgical outcomes and pathologic examinations}

Fifty five (54\%) lobectomies, 4 (4\%) bilobectomies and 45 (42\%) pneumonectomies were performed. A chest wall 
resection or extrapleural resection were performed in 29 (27\%) and 78 (73\%) patients, respectively. After the chest wall resection, large chest wall defects were repaired using a prosthesis in 14 patients. The operative mortality was $5 \%(n=5)$. Respiratory failure was the only cause of death. Perioperative complications occurred in 22 (21\%) patients. There were 12 pulmonary complications such as pneumonia or atelectasis. Among them, 6 patients suffered from acute respiratory distress syndrome (ARDS) which needed mechanical ventilator care. Bronchopleural fistula (BPF) and bleeding necessitating re-exploration occurred in one patient, respectively.

The mean tumor diameter was $6.2 \mathrm{~cm}$ (range, 2 to $15 \mathrm{~cm}$ ). There were 60 (56\%) squamous cell carcinomas and 25 (23\%) adenocarcinomas. In 67 (63\%) patients, tumoral invasion was limited to parietal pleura; in 40 (37\%) patients, it extended to the soft tissue or ribs. Pathologic stages were T3N0 in 64(60\%) cases, T3N1 in 19 (18\%) cases and T3N2 in 24 (22\%) cases. Complete resection was achieved in 90 (84\%) cases. Among R1 resection, there were 11 cases in the positive lateral resection margin and 5 cases in extracapsular invasion of retrieved lymph nodes. R2 resection occurred in 1 patient. Lymphovascular invasion was found in 18 patients. These results are summarized in Table 1.

Table 1 Demographic, surgical outcomes and pathologic features

\begin{tabular}{lc}
\hline & $\mathbf{N}=\mathbf{1 0 7}$ \\
\hline Age (years, median (range)) & $64.0(30-80)$ \\
Sex (male) & $91(85 \%)$ \\
FEV1 (liter, mean \pm SD) & $2.0 \pm 0.8$ \\
FEV1 (\%, mean \pm SD) & $76.5 \pm 25.9$ \\
Superior sulcus tumor & $17(16 \%)$ \\
Extent of resection & \\
$\quad$ Lobectomy & $58(54 \%)$ \\
$\quad$ Bilobectomy & $4(4 \%)$ \\
Pneumonectomy & $45(42 \%)$ \\
Type of resection & \\
$\quad$ Chest wall resection & $29(27 \%)$ \\
$\quad$ Extrapleural resection & $78(73 \%)$ \\
Mortality & $5(5 \%)$ \\
Tumor size (mean \pm SD) & $6.2 \pm 2.5$ \\
Depth of chest wall invasion & \\
Superficial invasion & $67(63 \%)$ \\
Deep invasion & $19(18 \%)$ \\
Nodal status & $24(22 \%)$ \\
N0 & $18(17 \%)$ \\
\hline Lymphovascular invasion & \\
\hline
\end{tabular}

FEV1; forced expiratory volume in 1 second

\section{Adjuvant treatments}

Among the 102 patients who did not die perioperatively, fifty one patients were referred for adjuvant chemotherapy. However, only 35 (69\%) patients completed adjuvant chemotherapy. In particular 19 patients (29.7\%) out of 64 patients with pT3N0 received adjuvant chemotherapy. Eight patients refused chemotherapy after one or two cycles of chemotherapy. During the $1^{\text {st }}$ or $2^{\text {nd }}$ chemotherapy, systemic metastasis was found in 5 patients. Treatment-related toxicity, such as pneumonia or BPF, occurred in 3 patients, causing a halting of any further treatment. Chemotherapy related mortality occurred in one patient due to pneumonia and septic shock. Postoperative radiotherapy was carried out in 51 (50\%) patients. Eighteen patients received postoperative chemoradiotherapy. No adjuvant treatment could be considered in 18 patients due to poor performance status and patient refusal.

\section{Recurrences and survivals}

Median follow up time was 16 months. Recurrences occurred in 54 patients. Eleven patients had only locoregional recurrences and 8 patients had loco-regional recurrences and systemic recurrences simultaneously. The locations of the systemic recurrences were as follows: Lung $(\mathrm{n}=15)$, brain $(\mathrm{n}=15)$, bone $(\mathrm{n}=11)$, liver $(n=5)$, adrenal gland $(n=3)$ and others $(n=4)$. Twenty nine patients are currently alive. Out of 78 patients who were not alive during the follow-up period, 46 patients (59\%) died within 1 year after the operation. The causes of 1 year mortality were as follows: cancer-related $(\mathrm{n}=$ $25,54 \%)$, pneumonia or respiratory failure $(n=16,35 \%)$, others $(n=3,7 \%)$ and unknown $(n=2,4 \%)$. One year mortality was higher in patients who underwent pneumonectomy compared to those who underwent lobectomy ( $57 \%$ vs $31 \%, p=0.011)$. The median survival was 15.9 months and overall 5 year survival rate was $26.3 \%$. According to the $\mathrm{N}$ status, 5 year survival was $37.4 \%$, $21.1 \%$ and $4.6 \%$ in cases of N0, N1, and N2 disease, respectively ( $p=0.047$, Figure 1$)$. The 5 year survival rate of patients with complete resection $(31.7 \%)$ was statistically higher than those with incomplete resection $(7.5 \%$, $\mathrm{p}<0.001$, Figure 2). The univariate prognostic factors for survival included gender $(p=0.029)$, extent of resection (pneumonectomy vs lobectomy, $p=0.041$ ), tumor size( $>$ $5 \mathrm{~cm}$ vs $\leq 5 \mathrm{~cm}, p=0.001$ ), nodal status (N0 or N1 vs $\mathrm{N} 2, p=0.029$ ), completeness of resection (complete vs incomplete, $p<0.001$ ) and completeness of adjuvant chemotherapy $(p<0.001)$. At multivariate analysis, five independent prognostic factors were shown; depth of invasion (superficial vs deep, $p=0.035$ ), tumor size ( $p=$ $0.007)$, nodal status $(p=0.001)$, completeness of resection $(p<0.001)$, and completeness of adjuvant chemotherapy $(p<0.001$, Table 2$)$. 


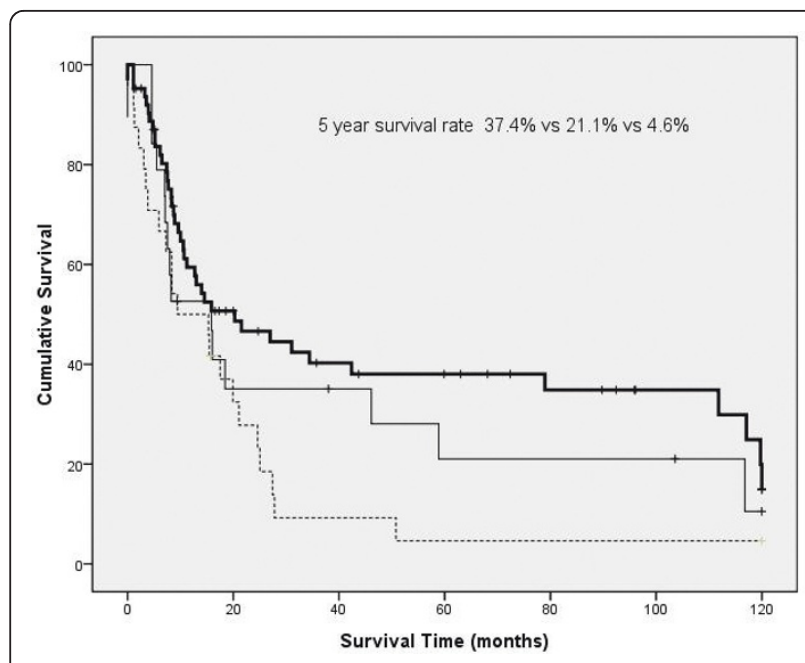

Figure 1 Survival curve according to node involvement (thick solid line: N0, solid line: N1, dotted line: N2).

5 year overall survival rates of patients with completion of adjuvant chemotherapy were statistically higher than those of patients without completion of adjuvant chemotherapy (5 year survival rate $46.7 \%$ vs $16.6 \%$ ). Statistical analysis for cancer-specific survival showed that the survival benefit was higher in the patient groups who received chemotherapy (5 year survival rate $45.8 \%$ vs $21.8 \%, p=0.001)$. And in patients with completely resected T3N0 NSCLC, the completion of chemotherapy is the only prognostic factor for long term survival ( 5 year survival rate $68.8 \%$ vs $29.7 \% p=0.011$, Figure 3 ) in univariate analysis. Our study also revealed that there was no statistical difference in survival with or without

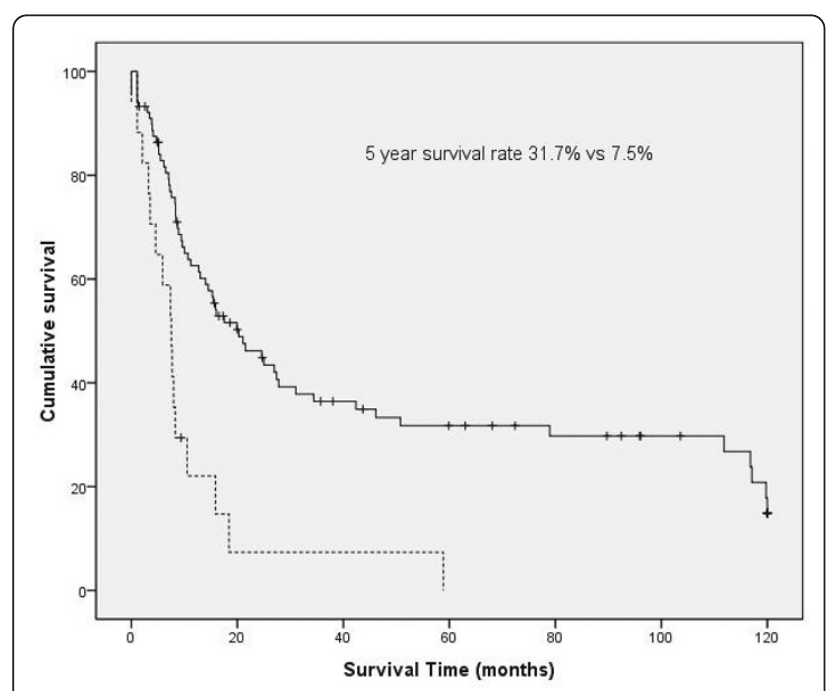

Figure 2 Survival curve according to completion resection (solid line: complete resection, dotted line: non complete resection) adjuvant radiotherapy (5 year survival rate $23.8 \%$ vs $29.9 \%, p=0.618)$.

\section{Discussion}

This study demonstrated that the long-term survival of patients with chest wall invading NSCLC were independently related to not only nodal status and R0 resection which have been consistently reported as prognostic factors, but also the depth of invasion, tumor size and adjuvant chemotherapy.

Since technical feasibility and the long-term survival of the surgically resected NSCLC invading chest wall were reported in 1947 [14], surgical resection has been the key component in the management of most patients with chest wall invading NSCLC. Except for the incompleteness of resection and the presence of lymph node metastasis, other factors influencing on survival are still unclear. In particular, controversies remain as to how extensive a resection should be $[7-9,15,16]$ and on the role chemotherapy or radiotherapy $[5,6,12,13]$.

Mean FEV1 of the patients enrolled in this study (76.5\%) was significantly lower than that of the patients in the total cohort (92.1\%) and this is thought to be the reason for high incidence of death due to pneumonia during the follow up period.

We performed a pneumonectomy in cases of large tumor that invaded to fissure and adjacent lobe. Relatively high percentage of pneumonectomy (42\%) was performed compared to previous studies that reported percentage of $20-27 \%[7,8,10]$. In the patients with tuberculosis and other inflammatory lung disease which were frequent in Korea, pneumonectomy was performed in cases large tumor crossed over major fissure and remnant lung volume is not enough. That's the reason why the incidence of pneumonectomy was high. Larger tumor size and high incidence of respiratory related mortality seemed to be attributable to significantly higher 1-year mortality of the patients who received pneumonectomy. Hence, pneumonectomy should be avoided in patients with chest wall invasion.

As previously reported [7-9], R0 resection and the absence of N2 disease was revealed as better prognostic factors for long term survival of patients with chest wall invading NSCLC in this study.

The prognostic significance of the degree of the chest wall invasion was not reflected in the $7^{\text {th }}$ TNM classification but the sub-classification of the T3 stage and prospective data collection were recommended for future revision [17]. There are several studies $[1,7,8]$ including ours, that report the better prognosis of a patient with tumor invasion in the parietal pleura compared to deep invasion. In case of superficial invasion, extrapleural resection is thought to be an adequate treatment option, considering the fact that there were 
Table 2 Univariate and multivariate analysis for long term survivals

\begin{tabular}{|c|c|c|c|c|c|c|c|c|}
\hline & \multicolumn{4}{|c|}{ Univariate analysis } & \multicolumn{4}{|c|}{ Multivariate analysis } \\
\hline & \multirow[t]{2}{*}{$p$ value } & \multirow[t]{2}{*}{ Odds ratio } & \multicolumn{2}{|c|}{$95 \% \mathrm{Cl}$} & \multirow[t]{2}{*}{$p$ value } & \multirow[t]{2}{*}{ Odds ratio } & \multicolumn{2}{|c|}{$95 \% \mathrm{Cl}$} \\
\hline & & & lower & upper & & & lower & uppe \\
\hline Sex (male/female) & 0.029 & 2.10 & 1.01 & 4.37 & 0.270 & 1.59 & 0.70 & 3.61 \\
\hline $\operatorname{Age}(\leq 65 />65))$ & 0.278 & 0.78 & 0.50 & 1.22 & NA & NA & NA & NA \\
\hline Extent of resection (pneumonectomy/lobectomy) & 0.041 & 1.59 & 1.02 & 2.49 & 0.410 & 1.24 & 0.75 & 2.06 \\
\hline Type of resection (Extrapleural resection vs chest wall resection) & 0.145 & 0.70 & 0.43 & 1.13 & 0.257 & 1.42 & 0.77 & 2.62 \\
\hline Tumor size $(\leq 5 \mathrm{~cm} />5 \mathrm{~cm})$ & 0.001 & 0.44 & 0.27 & 0.71 & 0.007 & 0.46 & 0.27 & 0.81 \\
\hline Lymphovascular invasion (no/yes) & 0.151 & 0.66 & 0.38 & 1.16 & 0.126 & 0.62 & 0.34 & 1.14 \\
\hline N2 lymph node involvement (N0,1/N2) & 0.029 & 0.57 & 0.35 & 0.94 & 0.000 & 0.34 & 0.19 & 0.61 \\
\hline Roresection (R0/R1,2) & 0.000 & 0.32 & 0.18 & 0.56 & 0.000 & 0.26 & 0.13 & 0.49 \\
\hline Depth of invasion (superficial/deep) & 0.148 & 0.72 & 0.46 & 1.12 & 0.035 & 0.56 & 0.33 & 0.96 \\
\hline Superior sulcus tumor (no/yes) & 0.939 & 1.02 & 0.58 & 1.83 & NA & NA & NA & NA \\
\hline Completion of chemotherapy (no/yes) & 0.000 & 3.18 & 1.86 & 5.41 & 0.000 & 4.83 & 2.47 & 9.43 \\
\hline Radiotherapy (no/yes) & 0.618 & 0.89 & 0.57 & 1.40 & NA & NA & NA & NA \\
\hline
\end{tabular}

NA; not available

no significant differences in the 5-year survival between the patients with parietal pleural invasion $(\mathrm{N}=67)$, who received extra-pleural resection $(\mathrm{N}=57)$ and chest wall resection $(\mathrm{N}=10)(29.7 \%$ vs $23.3 \%, \mathrm{p}=0.832)$, and the high incidence of complications among the patients who received chest wall resection $[8,11]$. In cases of deep invasion, moreover, there are no statistical differences between group of extrapleural resection and group of chest wall resection in terms of locoregional recurrence rates and 5 year overall survival rates if $\mathrm{R} 0$ resection was achieved.

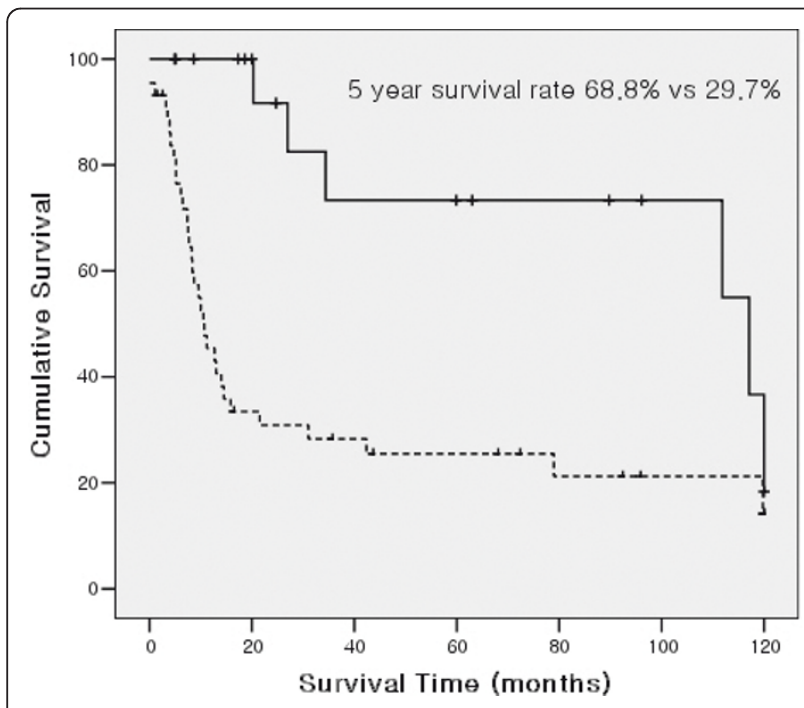

Figure 3 Survival curve according to completion of chemotherapy in patients with completely resected T3NO NSCLC (solid line: completion group, dotted line: non completion group)
Unlike the $6^{\text {th }}$ edition of the TNM staging, the T staging was sub-classified as $3 \mathrm{~cm}, 5 \mathrm{~cm}$ and $7 \mathrm{~cm}$ according to the tumor size in the $7^{\text {th }}$ edition of the TNM staging [17], and what it implicates is that the tumor size is an independent prognostic factor of NSCLC. The results of our study also show that the tumor size independently influences the prognosis of the NSCLC patient with chest wall invasion similar to the in other study [10].

The role of adjuvant chemotherapy in patients with completely resected NSCLC has been evaluated in many randomized clinical trials and meta-analysis. Cancer Care Ontario and American Society of Clinical Oncology concluded that adjuvant cisplatin-based chemotherapy is recommended for routine use in patients with stages IIA, IIB according to the $6^{\text {th }}$ edition of TNM Classification of Malignant Tumors [12]. However, De Pas et al [13] have asserted that the efficacy of adjuvant chemotherapy should be assessed separately in the T1-2N1 and T3N0 subgroups considering the fact that the efficacy of adjuvant chemotherapy usually varies according to lymph node status [14] and differences in biological behavior between subgroups. And they recommended that a pooled-analysis of the existing data would quickly and with limited effort provide a preliminary answer. Our result demonstrated that systemic recurrence occurred in about $80 \%$ of patients whom recurrences had occurred in, and adjuvant chemotherapy was also effective in terms of long-term survival especially in completely resected T3N0 patients. The cancer- specific survival was calculated because there was a subset of patients who could not receive chemotherapy due to their poor performance status and died of reasons such as pneumonia. Statistical analysis showed that the survival benefit was higher in the patient groups who received chemotherapy. These results implicate the possible benefit for 
postoperative chemotherapy for NSCLC patients with chest wall invasion although all confounding comorbidities were not completely reflected in cancer-specific survival. However, the fact that only 35 patients out of 107 patients, enrolled in this study, completed chemotherapy suggests the likelihood of preoperative chemotherapy in terms of expansion of delivery rate in definite preoperative chest wall invading T3 patients and the necessity of research in this area.

The role of adjuvant radiotherapy in patients with chest wall invading NSCLC is still controversial. Although two reports $[15,18]$ about beneficial effect of adjuvant radiotherapy have been published, other series $[2,5,7,19]$ have not showed any improvement in survival with use of postoperative radiotherapy. Our study also revealed that there was no statistical difference in survival with or without adjuvant radiotherapy.

The proposed strategy based upon prognostic factors mentioned above are as follow: Mediastinoscopy should be done irrespective of the chest CT or PET-CT results and neoadjuvant chemotherapy should be initiated if N2 is confirmed. Neoadjuvant chemoradiotherapy should be considered if the resection margins are expected to be insufficient due to large tumor size or deep invasion in the posterior location like Pancoast tumor [20,21]. Pneumonectomy should be avoided whenever possible because not only cancer related death but also respiratory related death is significantly high in this group of patients. If the tumor invades only the parietal pleura and separates without any remaining tumor tissue, an extrapleural resection could be done. Otherwise, chest wall resection should be performed to secure the wide resection margin. Postoperative adjuvant chemotherapy could be considered in patients with completely resected T3N0 chest wall invading tumors.

\section{Limitations}

A small number of patients and the retrospective method of reviewing the data remain as limitations of the study. The same protocol was not applied because the data were collected from a long period of time. However, mediastinal lymph node dissection was performed and a platinum based chemotherapy agent was used in all patients and hence, treatment strategy was relatively consistent.

Patients with a superior sulcus tumor were included in this study, unlike many other studies, that exclude them due to different behaviors of the disease itself. However, patients with a superior sulcus tumor invading the structure beyond the thorax were excluded in this study and the influence of the included patients with superior sulcus tumor might be small because the survival differences between patients with superior sulcus tumor and those with non superior sulcus tumor were insignificant.
Long term survival benefit of adjuvant chemotherapy after lung cancer surgery is known to be approximately $5 \%$, whereas the 5 year survival benefit in this study exceeded $30 \%$. This result seems to be the influence of patients whose postoperative performance was poor, included in the group of patients who did not receive chemotherapy. Hence, we calculated the cancer-specific survival rate but we think that this was not enough to completely exclude the effect of selection bias as well. Accordingly, this study has a hypothesis generating effect regarding the role of chemotherapy in lung cancer patients with chest wall invasion.

\section{Conclusion}

Completeness of resection, nodal status, depth of invasion, tumor size, and adjuvant chemotherapy were prognostic factors for long-term survival in NSCLC patients with chest wall invasion. Because of poor prognosis in cases with chest wall invasion that have $\mathrm{N} 2$ positive $\mathrm{LN}$, that is difficult to achieve complete resection and that need pneumonectomy, definite chemoradiotherapy or neoadjuvant chemoradiotherapy should be considered first in these cases. Further verification is needed through a larger group study due to the small sample size and retrospectively reviewed data.

\section{Acknowledgements}

This study was supported by a grant of the Korea Healthcare technology R\&D Project, Ministry for Health, Welfare \& Family Affairs, Republic of Korea. (A101211)

\section{Author details}

'Department of Thoracic \& Cardiovascular Surgery, Seoul National University Hospital, Seoul National University College of Medicine. ${ }^{2}$ Department of Internal Medicine, Yonsei University College of Medicine, Seoul National University Hospital, Seoul National University College of Medicine. ${ }^{3}$ Department of Thoracic and Cardiovascular Surgery, Seoul National University Hospital, Seoul National University College of Medicine.

\section{Authors' contributions}

CYL: Conception and design, drafting the article, final revision, CSB: Acquisition of data, interpretation of data, JGL: Revision for important content, DJK: Interpretation of data, revision for important content, BCC: Interpretation of data, revision for important content, KYC: Acquisition of data, interpretation of data, revision, IKP: Conception and design, revision, final approval. All authors read and approved the final manuscript.

\section{Competing interests}

Drs. Lee CY, Byun CS, Lee JG, Kim DJ, Cho BC, Chung KY and Park IK have no conflicts of interest or financial ties to disclose.

Received: 21 September 2011 Accepted: 12 January 2012

Published: 12 January 2012

\section{References}

1. McCaughan BC, Martini N, Bains MS, McCormack PM: Chest wall invasion in carcinoma of the lung. Therapeutic and prognostic implications. J Thorac Cardiovasc Surg 1985, 89:836-841.

2. Piehler JM, Pairolero PC, Weiland LH, Offord KP, Payne WS, Bernatz PE: Bronchogenic carcinoma with chest wall invasion: factors affecting survival following en bloc resection. Ann Thorac Surg 1982, 34:684-691. 
3. Trastek VF, Pairolero PC, Piehler JM, Weiland LH, O'Brien PC, Payne WS, Bernatz PE: En bloc (non-chest wall) resection for bronchogenic carcinoma with parietal fixation. Factors affecting survival. J Thorac Cardiovasc Surg 1984, 87:352-358.

4. Ricci C, Rendina EA, Venuta F: En bloc resection for T3 bronchogenic carcinoma with chest wall invasion. Eur J Cardiothorac Surg 1987, 1:23-28.

5. Allen MS, Mathisen DJ, Grillo HC, Wain JC, Moncure AC, Hilgenberg AD: Bronchogenic carcinoma with chest wall invasion. Ann Thorac Surg 1991, 51:948-951.

6. Pitz CC, Brutel de la Rivière A, Elbers HR, Westermann CJ, van den Bosch JM: Surgical treatment of 125 patients with non-small cell lung cancer and chest wall involvement. Thorax 1996, 51:846-850.

7. Downey RJ, Martini N, Rusch WW, Bains MS, Korst RJ, Ginsberg RJ: Extent of chest wall invasion and survival in patients with lung cancer. Ann Thorac Surg 1999, 68:188-193.

8. Magdeleinat P, Alifano M, Benbrahem C, Spaggiari L, Porrello C, Puyo P, Levasseur $P$, Regnard JF: Surgical treatment of lung cancer invading the chest wall: results and prognostic factors. Ann Thorac Surg 2001, 71:1094-1099.

9. Matsuoka H, Nishio W, Okada M, Sakamoto T, Yoshimura M, Tsubota N: Resection of chest wall invasion in patients with non-small cell lung cancer. Eur J Cardiothorac Surg 2004, 26:1200-1204.

10. Doddoli C, D'Journo B, Le Pimpec-Barthes F, Dujon A, Foucault C, Thomas $P$, Riquet M: Lung cancer invading the chest wall: a plea for enbloc resection but the need for new treatment strategies. Ann Thorac Surg 2005, 80:2032-2040.

11. Kawaguchi K, Mori S, Usami N, Fukui T, Mitsudomi T, Yokoi K: Preoperative evaluation of the depth of chest wall invasion and the extent of combined resections in lung cancer patients. Lung Cancer 2009, 64:41-44.

12. Pisters KM, Evans WK, Azzoli CG, Kris MG, Smith CA, Desch CE, Somerfield MR, Brouwers MC, Darling G, Ellis PM, Gaspar LE, Pass HI, Spigel DR, Strawn JR, Ung YC, Shepherd FA: Cancer Care Ontario and American Society of Clinical Oncology adjuvant chemotherapy and adjuvant radiation therapy for stages I-IIIA resectable non small-cell lung cancer guideline. J Clin Oncol 2007, 25:5506-5518.

13. Pas TD, Raimondi S, Pelosi G, Spaggiari L, Braud FD, Veronesi G, Maisonneuve P: A critical appraisal of the adjuvant chemotherapy guidelines for patients with completely resected T3NO non-small-cell lung cancer. Acta Oncol 2010, 49:480-484.

14. Douillard JY, Rosell R, De Lena M, Carpagnano F, Ramlau R, GonzálesLarriba JL, Grodzki T, Pereira JR, Le Groumellec A, Lorusso V, Clary C, Torres AJ, Dahabreh J, Souquet PJ, Astudillo J, Fournel P, Artal-Cortes A, Jassem J, Koubkova L, His P, Riggi M, Hurteloup P: Adjuvant vinorelbine plus cisplatin versus observation in patients with completely resected stage IB-IIIA non-small-cell lung cancer (Adjuvant Navelbine InternationalTrialist Association [ANITA]): A randomized controlled trial. Lancet Oncol 2006, 7:719-727.

15. Patterson GA, Ilves R, Ginsberg RJ, Cooper JD, Todd TR, Pearson FG: The value of adjuvant radiotherapy in pulmonary and chest wall resection for bronchogenic carcinoma. Ann Thorac Surg 1982, 34:692-697.

16. Coleman FP: Primary Carcinoma of the Lung with Invasion of the Ribs: Pneumonectomy and Simultaneous Block Resection of the Chest Wall. Ann Surg 1947, 126:156-168.

17. Peter G: Staging manual in thoracic oncology. Florida: An International association for the study of lung cancer publication; 2009, 57-89.

18. Facciolo F, Cardillo G, Lopergolo M, Pallone G, Sera F, Martelli M: Chest wall invasion in non-small cell lung carcinoma: a rationale for en bloc resection. J Thorac Cardiovasc Surg 2001, 121:649-656.

19. Burkhart HM, Allen MS, Nichols FC, Deschamps C, Miller DL, Trastek VF, Pairolero PC: Results of en bloc resection for bronchogenic carcinoma with chest wall invasion. J Thorac Cardiovasc Surg 2002, 123:670-675.

20. Rusch WW, Giroux DJ, Kraut MJ, Crowley J, Hazuka M, Winton T, Johnson DH, Shulman L, Shepherd F, Deschamps C, Livingston RB, Gandara D: Induction chemoradiation and surgical resection for superior sulcus non-small-cell lung carcinomas: long-term results of Southwest Oncology Group Trial 9416 (Intergroup Trial 0160). J Clin Oncol 2007, 25:313-318.

21. Kunitoh H, Kato H, Tsuboi M, Shibata T, Asamura H, Ichonose $Y$, Katakami $N$, Nagai K, Mitsudomi T, Matsumura A, Nakagawa K, Tada H, Saijo N, Japan Clinical Oncology Group: Phase II trial of preoperative chemoradiotherapy followed by surgical resection in patients with superior sulcus non- small-cell lung cancers: report of Japan Clinical Oncology Group trial 9806. J Clin Oncol 2008, 26:644-649.

doi:10.1186/1477-7819-10-9

Cite this article as: Lee et al:: The prognostic factors of resected nonsmall cell lung cancer with chest wall invasion. World Journal of Surgical Oncology 2012 10:9.

\section{Submit your next manuscript to BioMed Central and take full advantage of:}

- Convenient online submission

- Thorough peer review

- No space constraints or color figure charges

- Immediate publication on acceptance

- Inclusion in PubMed, CAS, Scopus and Google Scholar

- Research which is freely available for redistribution 